\title{
Strengthening social ties via ICT in the organization
}

\author{
Osku Torro \\ Department of Information Management and \\ Logistics \\ Tampere University of Technology \\ osku.torro@tut.fi
}

\author{
Henri Pirkkalainen \\ Department of Information Management and \\ Logistics \\ Tampere University of Technology \\ henri.pirkkalainen@tut.fi
}

\begin{abstract}
Knowledge work increasingly relies on the utilization of information and communication technology (ICT). However, communication and knowledge sharing via ICT may be challenging due the lack of physical face-to-face interaction. The strength of social ties is critical to the success of an organization, since it determines how deeply individuals interact with each other. Prior research has paid only limited attention to the role of ICT in the strengthening of social ties within an organization. To address this research gap, we have conducted a qualitative study outlining different tie strengthening characteristics of ICT. The results of this study suggest that especially asynchronous and synchronous text based interaction and communication history forms an effective mechanism for an organization to facilitate social ties. As a theoretical contribution, we develop a new theoretical model representing the intraorganizational characteristics of ICT in relation to media synchronicity and tie strength. This theoretical model also includes new tie-strength components for ICT-mediated interaction.
\end{abstract}

\section{Introduction}

Information and communication technology (ICT) has helped people communicate with each other more easily today than at the beginning of the millennium. Rapidly evolving technology reveals new capabilities for organizations [1]. At the same time, the nature of work, particularly in the context of knowledge work, has changed substantially: physical presence is no longer necessary, and distributed work has become a viable option [1-3]. ICT-mediated work, such as using different collaboration technologies, has a considerable impact on organizations' performance [2-4].

However, managing ICT-mediated interaction is far more challenging than traditional face-to-face work $[5,6]$. Addressing these challenges is a critical factor for organizational success in today's complex and turbulent business environment [4, 7]. Creating personal relationships (i.e. social ties) in ICTmediated work is difficult because of the lack of physical human interaction. This in turn affects how teams perform and how individuals carry out their professional duties [7-9]. Strong social ties also positively affect information transfer and adoption in the organization [10-12], hence, it has a major impact on the overall effectiveness of organizations.

It is recommended that the organization should build social relationships by using ICT [5]. However, the literature does not provide a clear answer on the roles and specific characteristics of different ICTs in building these relations. There is not enough research on social tie strengthening in organizational contexts (excluding trust, as discussed later in this article), particularly if interaction occurs via ICT. Also, many attempts have been made to find valid tie-strength components for measuring social ties [13], but it is not well understood how ICT-mediated interaction affects to the actual initial setting. There is a need to analyze the most relevant tie-strength components behind different levels of ICT-mediated interaction.

Given the scope of the research gap, our aim is to uncover and explain the key factors behind building social relations via ICT in an organization. The main research question is: what characteristics of ICTmediated interaction strengthen social ties within an organization? The research question is first approached through a literature review based on Granovetter's [14] theory of weak and strong ties. We conducted a qualitative study that builds on the theoretical foundation set by Granovetter [14], and other significant social ties related research found in literature [11, 13]. This study uncovers a previous unmapped area of tie strengthening via ICT, focusing especially on knowledge work in organizations. The data was gathered by means of theme interviews and analyzed using qualitative content analysis $[15,16]$.

The study found that it is possible to strengthen social ties in organizations at various levels. These levels are presented in the theoretical model, which 
forms three main categories: (1) asynchronous and (2) synchronous text-based interaction and (3) VoIP/video-based interaction. These levels of interaction are modified due analysis of the data, and extended from media synchronicity theory (MST) [17]. The interface (i.e. the shared boundary) between asynchronous and synchronous levels of this theoretical model was particularly important when strengthening social ties in the organization.

This study makes several contributions to tiestrength research. First, it presents a new theoretical model to explain intra-organizational ICT characteristics in relation to media synchronicity and tie strength. Second, the study illustrates different levels of ICT-mediated interaction and their relation to tie-strengthening. Finally, the presented model connects several (new and previously identified) tiestrengthening components to different technological characteristics. As a practical contribution, this study elaborates on the so-called "Sweet spot of tiestrengthening," which is the critical melting pot that organizations should strive for.

\section{Related research and Theoretical Background}

Weak and strong ties constitute part of Mark Granovetter's [14] sociological network theory in which he combined the relationships between individuals (micro level) to the structure of whole social networks (macro level). According to Granovetter [14], ties between people are either weak, strong, or absent. The strength of ties defines also the strength of relationships between individuals. Tie strength affects, for example, how information is transferred and adopted in the social network [14].

\subsection{Weak and strong ties}

According to Granovetter [14], it can be intuitively concluded whether a given tie is strong, weak, or absent. Strong ties are formed between people that are close to us: family, friends and other people with whom we spend a lot of time, like colleagues. Weak ties are characterized that they are active only a short period of time and formed mainly between acquaintances or previously unknown individuals.

Granovetter [14] argues that the importance of strong ties has been overemphasized and that the importance of weak ties is not fully understood. Weak ties are more valuable because new information (idea, innovation, rumors, etc.) is transferred mainly through the network of weak ties. Weak ties can establish a relationship between strongly tied networks, for example, departments within an organization [3]. Information transferred through these weak ties, "local bridges," is diverse and less redundant due to the variety of specialized knowledge across different organizational units [10]. A large number of weak ties helps new information to migrate into the wider area [14, 18], however, a large number of weak ties in an organization is not an absolute value in itself. Their quality also matters, because information obtained through weak ties is not always fully usable or relevant, or willingness to share information might be low [2, 19-22]. The Internet enables an increasing number of weak tie networks between individuals, which can be managed by, for example, a layer of different social networking services $[3,9]$. However, weak ties might become dependent on the medium of the relationship $[3,18]$, which highlights the role of intraorganizational ICT in facilitating the network of weak ties.

Strong ties tend to bond similar or like-minded people to each other and to form high-cohesion, mutually-connected networks $[11,12,14]$. Therefore, the information contained within networks consisting of strong ties is usually highly redundant, and the knowledge adoption of both explicit and tacit knowledge tends to be high [12, 19]. Organizational networks consisting of strong ties are considered essential when dealing with complex information [10]. Informal, strong ties ("friendship") help with transferring and adopting information in an especially effective way, mainly through higher levels of trust and mutual understanding. In addition, the devotion to others provide motivation for knowledge sharing [11]. Informal communication supports formal networks by exposing participants to relationships with people who have different kinds of information than they do. [23]. Similarly, the "weakness" of strong ties is correlated with lower information transfer and adoption [11].

Strong ties are good at facilitating change, providing socioemotional support and solving conflicts $[10,11]$. They tend to be independent of organizationally established media since willingness to interact via different technologies is higher than between weak ties. Therefore, strong ties are more robust under changing conditions [18].

\subsection{Strengthening ties via ICT}

The strengthening of social ties within an organization has received relatively little research attention. There are even less studies on the strengthening of social ties via ICT-mediated interaction. Previous research covers, for example, 
the meaning of social ties in virtual collaboration, transferring knowledge, or building social capital [6, 9, 12]. Moreover, the building of personal relationships via ICT has been broadly examined for example, in forming trust [24, 25] - but there is an absence of research on actual social tie strengthening via ICT. Trust has an essential role in tie-strengthening, but it is only one of the many tiestrength components [13, 14].

In Granovetter's [14] theory, there are four identifying properties of a strong tie: "The strength of a tie is (probably a linear) combination of the amount of time, the emotional intensity, the intimacy (mutual confiding), and the reciprocal services which characterize the tie" [14]. Krackhardt [11] points out that only the first one, amount of time, can be objectively measured. Moreover, the binary nature of tie strength generates problems in attempts to measure it objectively: at what point does the weak tie become strong? Tie strength is categorically either weak or strong, even though measurement methods are subjective (the emotional intensity, the intimacy, the reciprocal services) and linear (the amount of time).

Due to these challenges, labels such as "friend" and "colleague" have been used to describe the nature of strong ties [11]. Regular contact with a colleague forms, by definition, a strong tie, but it is obvious that relationships between colleagues and friends differ from each other. Therefore, in practice, how tie strength ultimately influences individuals' actions also depends on the nature of the relationship.

Measuring tie strength has proven to be difficult because of the lack of coherent measurement methods. The literature contains many different tiestrengthening components, but it is not known how well the tie-strength components found in the literature react in online environments [13]. Moreover, measuring tie strength seems to be highly dependent on subjective interpretation.

It also appears obvious that the role of technology must be taken into account. The actual strengthening of ties via ICT can only be studied if the most relevant tie-strength components can be connected to the characteristics of technology. In his original article, "The Strength of Weak Ties," Granovetter [14] states that "The fundamental weakness of current sociological theory is that it does not relate micro-level interactions to macro-level patterns in any convincing way." Extending Granovetter's [14] theory to ICT-mediated interaction leads to a very similar situation. The impact of separate technologies and software ("micro-level") should be explored in a coherent manner so that their impact can be monitored from the perspective of the entire network ("macro-level"). This would provide a deeper, more comprehensive understanding of how to facilitate and enhance intra-organizational relationships via ICTmediated interaction.

\section{Method}

We conducted a qualitative study through the collection of interview data, which was analyzed using qualitative content analysis [15, 16]. This approach was chosen because qualitative methods are useful in seeking to provide rich and immersive theoretical analyses of previously unmapped phenomena. [16, 26].

\subsection{Data collection}

The data comprises a total of 20 interviews from 12 different organizations with Finnish knowledge workers. The sampling method was purposeful in order to select information-rich participants. Snowball sampling was used to identify additional respondents [27]. To gather diverse information about the phenomenon, data was gathered from both ICT researchers $(6 / 20)$ and private sector employees (14/20). However, a strict focus on intraorganizational ICT-mediated knowledge work was maintained during the data gathering process. The interviews were semi-structured [16] and lasted from 30 to 60 minutes, resulting approximately 14 hours of taped recordings.

Basing on the theoretical definition of strong and weak ties, we first asked questions about the usage of ICT in relation to different relationships within an organization (i.e., strangers, acquaintances, colleagues, friends, etc.). We then moved to more detailed questions about the characteristics of ICT and how interviewees experienced ICT-mediated interaction within an organization. To ascertain the most relevant aspects of the phenomenon and to exploit the considerable amount of ICT-related practical expertise and theoretical knowledge that the interviewees possessed, means of appreciative inquiry were also applied. Schultze and Avital [28] maintain that "an appreciative interview is particularly appropriate in studies that aim to surface and capitalize on the capacity of people, teams, and organizations to construct enriching practices, and design work environments through a discourse that encourages positive change and participative action." 


\subsection{Data analysis}

The data was analyzed using qualitative content analysis [15, 16], which was undertaken throughout the data gathering process. A research diary and research notes helped to refine the data during the analysis. The process was iterative; the theoretical background was occasionally reviewed in order to better understand the phenomenon. As the data gathering process matured, it began to appear clearer that the tie-strength literature did not provide sufficient tools to analyze tie strengthening via ICTmediated interaction. Petróczi et al. [13] have gathered the most relevant tie-strengthening components together, but the analysis of the data suggested that other components also exist in ICTmediated interaction.

Tie-strength components were the central part of the coding frame used in the analysis and were compared to the different levels of interaction. Therefore, as the tie-strength components were customized to fit the research question, the coding frame of the data analysis was also modified. After the completion of the new coding frame, the data was re-analyzed in a highly systematic manner. At this point, the speed of the data collection was significantly enhanced. With the help of new tiestrength components, over 300 quotations were gathered, and the content began to reach saturation point.

\section{Results}

It is possible to strengthen social ties in organizations at various levels of interaction on the basis of different technological characteristics. These characteristics form three main levels of interaction: (1) asynchronous and (2) synchronous text-based interaction and (3) VoIP/video-based interaction. We built a theoretical model of tie strengthening via ICT that combines the central results of the data-driven analysis (Figure 1.)

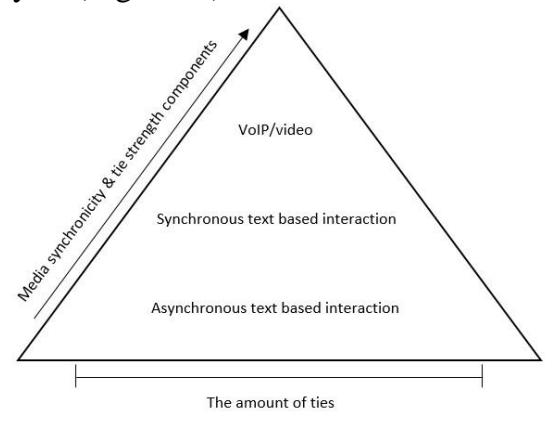

Figure 1. Theoretical model of tie-strengthening via ICT
The ingredients of this theoretical model are presented in Table 1. The levels of interaction are modified due analysis of the data, and extended from media synchronicity theory (MST) [17]. Tie-strength components are based on the analysis of the data, or extended from tie-strength literature [13]. Based on the analysis of the data, both media synchronicity and tie strength are closely connected to the different levels of interaction as well as the number of potential ties.

This model is intended to provide an approach for understanding tie strengthening via ICT in organizational settings. We now discuss the results in relation to the three levels of interaction. These sections discuss the relevant tie-strengthening components through examples from our data.

\subsection{Asynchronous text based interaction}

The most relevant tie-strength components at asynchronous text-based interaction are the fit of media, reciprocity, spontaneity, informality, and interpersonal trust.

The network of weak ties was highlighted at this level of interaction. Strong ties were maintained mainly through specific task-related channels or social networking tools. This type of communication was highly formal. Informal communication occurred mainly through social networking services, but otherwise, it was mainly absent.

Some interviewees had a strong preference for the use of asynchronous channels, especially email. It was emphasized that emails allowed carefully detailed information changes, which was preferred if information was of a personal nature or task-related context. If the shared information was relevant to the entire network, other asynchronous tools, such as social networking services, were preferred. For some individuals, interaction via asynchronous channels felt more comfortable, even compared with face-toface interaction, thus resulting in better communication performance.

There is this silent fellow... He does not say anything. But he's really good with email.

The transparent nature of different collaboration tools and social software and, for example, the ability to assign and take responsibility for tasks increased reciprocity. When needed, both task-oriented and company-wide asynchronous channels provided help to achieve tasks: 
Table 1. The ingredients of theoretical model

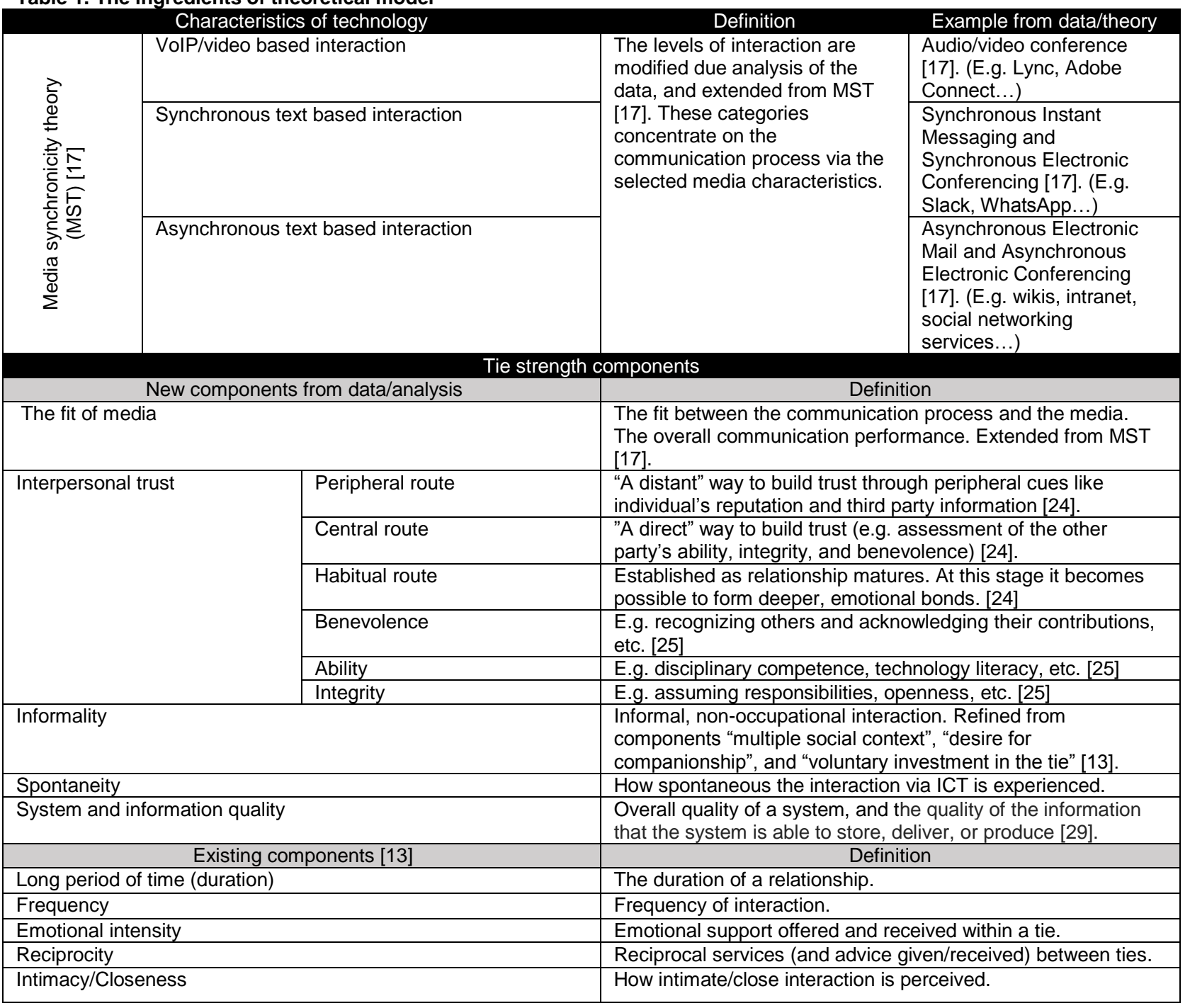

It's important to have a transparent workflow management system, so it'd visible if there's a task that's not progressing. I might notify someone, 'there's a task hanging, didn't you notice it, do you need help'?

It is easy to begin collaboration, if I notice from [company] wiki that someone has asked about this particular subject.

Asynchronous information transfer combined with the transparency of information (for example, status notifications, calendar outlook, etc.) also increased spontaneity. It thus felt easier and "less intrusive" to contact another person.

In particular, considerable amounts of information transfer occurred with the help of organizational social networking services, which enabled high levels of both formal and informal communication. Formal channels provided disciplinary information and knowledge while informal communication helped individuals understand each other better as persons. The communication history (formal and informal) of asynchronous text-based interaction helped to leverage the strengthening of social ties:

Especially for those who are new to the organization, it's great to see the communication history [of Yammer discussions]. There was a new member on the board of directors; it really helped her learn the firm's culture and history.

Every tool is used to both formal and informal [communication]. Only the ratio is changed.

The visible communication history in the organization sped up the trust-forming process from the peripheral to the central route. Informal communication was also seen as essential for building benevolence-based trust. Moreover, both benevolence- and ability-based trust were formed through the central route when individuals were able to express their disciplinary competences or personal characteristics in written, carefully detailed form: 
You'll use more time to write messages [email] that describe both you as a person and the context of an issue.

In addition, system and information quality leveraged trust formation: email encryptions and digital signatures helped to form trust through the central route. This was also the case for email integration, for example, LinkedIn profiles leveraged trust formation through the peripheral route.

\subsection{Synchronous text based interaction}

Synchronous text-based interactions were used in a versatile manner to maintain and strengthen both weak and strong ties. However, the role played by strong ties was significantly greater than at the asynchronous level of the theoretical model. The most relevant tie-strength components at this level of the theoretical model were: the fit of media, spontaneity, frequency, interpersonal trust, informality, and emotional intensity.

The fit of media for the context and its ability to transfer task-related information was highlighted:

I use Team Rooms in the Team Foundation Server, which is, in a way, the same as Slack, except integration with other software than TFS is not that good. I also use Skype for Business that has chatrooms, which are quite the same as TFS Team Rooms or Slack, which are, in turn, close to IRC-channels. IRC includes bots and integrations, but it's easier to export integrations from Slack. For example, all status changes from Trello are transported directly to Slack's event stream.

Although the information may be scattered in different task-related channels, synchronous textbased interactions also played an important role in streamlining the transfer of information in the organization.

The ability to create either permanent or ad hoc channels around specific themes or tasks was considered important. This also had a major boundary spanning effects on the organization. Members of different organizational units took part in different know-how teams built around specific themes of interest such as marketing, information security, and so on.

It seems also obvious that synchronous text based interaction allows tie strengthening via enhanced communication frequency and spontaneity. Synchronous text based interaction enabled "fast lane" to transfer information. However, meaning of less hectic, informal communication was also highly emphasized. Different chat and instant messaging tools replaced the lack of physical interaction. Accordingly, informal communication laid the foundation to the appearance of emotional intensity. Many interviewees considered so called "virtual coffee rooms" extremely important:

It's good to have a channel that is not workoriented. It reduces stress levels and improves coping at work. It's a virtual coffee room.

You can talk about your worries, house construction projects, and so on, at least with the people you know a little better.

Synchronous text-based tools enabled many possibilities to build trust: personal interaction via chat or instant messaging enabled the building of trust through the central route. "Virtual reputation" gained from, for example, organizations' public channels, built trust through the peripheral route.

[Previously unknown] people become more familiar when "echoing" themselves on the firm's public channels.

The habitual route to develop trust was also used because it was customary to interact with the same people via the same channels for a long time, even years. Building especially benevolence- and integrity-based trust was highly emphasized; tools were used to recognize team members, acknowledge their contributions, and express openness and involvement. Also, for some individuals, being socially active via chat or instant messaging was easier than face-to-face interaction.

One of the most important attributes of synchronous text-based interaction was, slightly paradoxically, its asynchronous nature. Organizations' formal and informal communication history formed an important mechanism for tie strengthening. It helped individuals observe interaction and adjust their own interaction to levels with which they felt personally comfortable:

Communication history should be visible. What has been discussed, what tasks has been closed. You see the whole [organizational] culture from a certain viewpoint. "How formal was this channel?" There are a couple of work-related channels and a "not safe for work" channel. If you view their history, you'll get access to the context [of information]. I might jump right in or just observe others. In a way, 
it's like driving to the motorway from a ramp; you adjust your own speed to that of others.

\subsection{VoIP/video based interaction}

The role of strong ties was significantly higher at this level of the theoretical model. Many interviewees avoided forming VoIP or video connections, especially those with weak ties. The most relevant tie-strength components at this level were: the fit of media, spontaneity, interpersonal trust, intimacy, and system and information quality.

Willingness to use VoIP or video connection varied significantly. Some interviewees felt that the amount of intimacy was excessive if communication occurred via VoIP or video connection. Those interviewees favored mainly text-based tools for interaction, especially between weak ties:

Having a Skype call with a previously unknown person... I would feel awkward.

No video [with strangers or acquaintances], it's too intimate.

VoIP or video calls were favored mainly for personal or group discussions between strongly tied individuals as a substitute to face-to-face meetings or as a quick and efficient way to transfer information. However, the ability to process information via VoIP or video connection was limited. Attempts to handle complex information were experienced as extremely challenging even if the ties between participants were strong.

It is possible to share tasks, you do this and I do that, but sharing different waypoints... that remains shallow.

However, complex information was successfully handled via VoIP or video in cases where, in addition to strong ties, regular physical interaction and/or high frequency interaction via various text-based tools occurred. This was experienced as particularly rewarding:

A customer requests some support, and we try to solve it with colleagues via Skype from different locations. When we finally succeed, everyone is happy. Then it's like "sociability++" for everyone.

None of the interviewees favored VoIP or video calls with previously unknown people. However, VoIP or video connection was used if it was felt necessary to form trust or express intimacy in an efficient way in situations such as important formal discussions or virtual groupings between previously unknown individuals. Forming benevolence and ability-based trust through the central route was highlighted. Besides the well-known importance of non-verbal communication, synchronicity itself leveraged trust building: there was no time to, for example, search elsewhere for relevant information.

Finally, the quality of the tools played an important role in transferring high amounts of media synchronicity. Low-quality tools created barriers to tie strengthening. Lack of usability decreased spontaneity. Connection lag and delayed responses, overlapping voices and low-quality video decreased communication performance; thus, many benefits of the media were diminished. Many of these issues made it challenging to maintain or strengthen social ties, especially in weakly tied networks.

\section{Discussion}

Despite the gap in the research, ICT-mediated interaction seems to be a very practical approach for studying the strengthening of social ties. It is the technological aspect itself that enables the opportunity to study the details of interaction more closely. Technology seems to acts as a "filter" so that the most relevant tie-strengthening components become more visible. This helps us to study the different characteristics of technology that leverage the strengthening of social ties in the organization.

Clearly, there are many ways of strengthening social ties via ICT, the most obvious being cases in which the use of technology is meant to substitute face-to-face interaction. High media synchronicity enables deeper interaction, especially between strong ties or on occasions when, for example, an effective way to form trust or transmit intimacy is needed. In terms of the tie-strengthening perspective, using VoIP or video connection between weak ties in casual or task-related routines is somehow seen as problematic as some individuals may consider the amount of intimacy excessive. VoIP or video connection is at its best between strong ties. If the ties are sufficiently strong, and the amount of redundant information sufficient, it might be possible to solve complex tasks via VoIP or video connection. If the ties between participants are too weak, the actual interaction via these tools remains shallow.

However, from an organizational point of view, the most interesting part of the theoretical model seems to be the interface (i.e. shared boundary) between asynchronous and synchronous interaction, which acts as a tie-strengthening "sweet spot" in the organization (Figure 2.): 


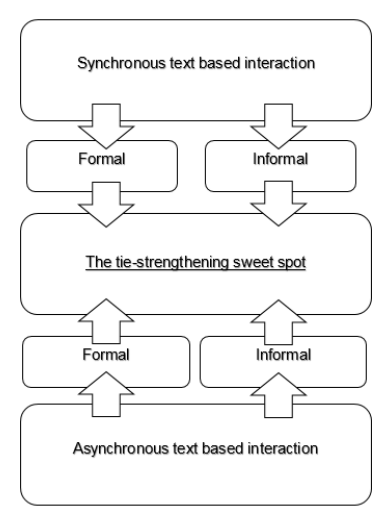

Figure 2. The sweet spot of the theoretical model

The interface between these two levels of interaction forms an area in the theoretical model in which asynchronous and synchronous text-based interaction and communication history create significant amounts of social and task-related activity in the organization. Tie-strengthening mechanisms at these levels of interaction are versatile, ranging from information transfer between weak ties to expressing emotional intensity between strong ties. The tiestrengthening sweet spot seems to play a particular role in channeling interaction between so-called intermediate (medium strong) ties in the organization. In fact, earlier studies suggest that intermediate ties are the dominant links among key organizational groups and that their actions should be supported by appropriate ICT [30]. The sweet spot of the theoretical model helps to reveal the technological characteristics of this "appropriate ICT" needed.

Earlier studies also reveal that intermediate ties are most effective in information transfer. Weak ties offer little chance of information transfer mainly due to the low frequency of interaction [31]. This justifies even further why the strengthening of social ties in the organization is needed. The sweet spot of the theoretical model facilitates relatively high amounts of interaction (most of the interviewees favored the combination of asynchronous and synchronous textbased interaction with weak and strong ties as well), so it seems safe to conclude that it offers an organization truly interesting possibilities in terms of building social relationships.

Whilst the ability to transform knowledge between organizational units is significantly important to organizational performance, such as capability to innovate, it is felt difficult to transfer especially deeper, contextual knowledge (e.g., deeper knowledge of others, being aware of progress on tasks...) or meta-knowledge (i.e. knowledge of who knows what, who knows whom) via ICT-mediated interaction. To overcome these challenges, previous research emphasizes, for example, the importance of communication visibility and access to different sources of information, like historical connections between entries $[4,12,32]$. This study has led to very similar findings: communication visibility [32] and access to different sources of information [4] is essential when increasing the capacity to transfer and adsorb information in the network. This is no wonder as the strength of ties is closely related to the amount of redundant information in the network [12, 14]. Thus, it seems obvious that the mechanisms that help an organization to strengthen the social ties, leverage also the building of meta-knowledge and contextualized knowledge. However, in this study, the viewpoint differs decisively from previous research. The tie-strengthening perspective tries to find the technological characteristics that build relationships via ICT-mediated interaction.

The role of informal interaction via ICT should also be underlined. It is clear that people are not only pragmatic; their decision-making is also affected by, among other things, emotions, values, and selfidentity [20]. Forming informal relationships in the organization also enhances the functioning of ICTmediated work. Strong informal ties help employees participate in the organization's activities in a meaningful way and to manage tasks efficiently and cooperatively in a virtual environment.

\subsection{Implications for theory}

This study uncovers a previously unmapped area of tie strengthening via ICT, focusing especially on knowledge work in organizations. The study contributes to research by developing a new theoretical model representing intra-organizational ICT characteristics in relation to media synchronicity and tie strength. The model illustrates different levels of ICT-mediated interaction and their relation to tiestrengthening. Another important contribution to research are tie-strengthening components customized to ICT-mediated interaction. This provides a coherent way of studying the tiestrengthening characteristics of technology rather than studying different technologies or software individually.

The theoretical model and the findings are the general approach to the phenomenon and are not to be interpreted blindly. A good deal of work is needed to refine this theory for a more detailed understanding. Because of the scope of the research gap, this study serves as a valid starting point for further theoretical discussion. 


\subsection{Implications for practice}

This study provides organizations with an understanding of the key characteristics behind technology that strengthen the social ties within organizations. This can help an organization to plan managerial actions (managing communication processes and performance rather than technology) and provide appropriate ICT tools to its employees so that the strengthening of social relationships can occur efficiently even in virtual environments. Providing appropriate tools is especially relevant when facilitating weakly tied networks since weak ties are more dependent on the medium of the relationship [3, 18]. The results of this study lead to the conclusion that both asynchronous and synchronous text-based tools are particularly effective in the strengthening of social ties to facilitate both ICT-mediated interaction and communication history within an organization. These tools help individuals manage tasks efficiently and cooperatively, and to interact informally, which is, at least in practice, necessary when forming stronger social ties.

While text-based interaction seems to act as a de facto standard for social tie strengthening in an organization, tools that provide higher media synchronicity, like VoIP or video, are particularly useful when building social relationships between strong ties. If the ties between individuals are sufficiently strong, and the amount of redundant information is sufficient, it might even be possible to solve complex problems via VoIP or video connection. Accordingly, text-based tools provide an excellent basis for regular interaction when it might be easier for individuals to absorb the usage of media with increased synchronicity, which, in turn, leverages even further the strengthening of social ties.

By providing the right tools for the right context, and understanding the possibilities and limitations of different ICTs, an organization is able to create a versatile network of ICT-mediated interaction that strengthens social ties between individuals in a diverse and effective way.

\section{Conclusion}

The study contributes to research by developing a new theoretical model that represents intraorganizational ICT characteristics in relation to media synchronicity and tie strength. The tiestrengthening characteristics of technology were more closely explored at three levels of ICTmediated interaction: (1) asynchronous and (2) synchronous text-based interaction and (3) VoIP/video-based interaction. The most relevant tiestrengthening components from each of these levels were presented.

The most interesting part of the theoretical model was the interface between asynchronous and synchronous interaction. The sweet spot of the theoretical model (Figure 2.) offered an organization an effective and versatile way of strengthening social ties from within. Higher levels of interaction (i.e., VoIP/video) strengthened social ties via increased amounts of media synchronicity. However, this was seen as problematic when interaction occurred between weak ties or in seeking to transfer complex information.

This study does have some limitations, many of which open up topics for future research. Importantly, one of the main goals of this study was to lay the foundation for further theoretical discussion. How can the theoretical model presented be applied outside knowledge work? In addition, the interviewees did not necessarily dismantle in detail topics related to, for example, emotional intensity, as they might have felt that this issue was overly personal. For potential future topics, we encourage researchers to study how ties are strengthened in different virtual communities. It is possible that those results could be applied to organizational settings. Also, many interviewees saw the possibilities of virtual and augmented reality for tie strengthening as very promising. Unfortunately, the amount of data did not give us the possibility for further analysis of this level of interaction and therefore this topic remains a topic for future research.

More in-depth analyses of the phenomenon are needed. Despite the limitations, this study helps us understand how social relationships could be built via ICT-mediated interaction within organizations.

\section{Acknowledgements}

This study was funded by the Academy of Finland grant \#259831.

\section{References}

[1] C., Forman, J. L. King, K. Lyytinen, "Special Section Introduction-Information, Technology, and the Changing Nature of Work", Information Systems Research, 2014, 25(4), pp. 789-795.

[2] T. Griffith, J. Sawyer, and M. Neale, "Virtualness and knowledge in teams: Managing the love triangle of 
organizations, individuals, and information technology", Mis Quarterly, 2003, 27(2), pp. 265-287.

[3] B. R. Katzy, C. J. Stettina, L. P. J. Groenevegen, M. J. de Groot, "Managing weak ties in collaborative work", $17^{\text {th }}$ international conference on concurrent enterpricing (ICE), 2011.

[4] X. Zhang, V. Venkatesh, and S. Brown. "Designing Collaborative Systems to Enhance Team Performance",

Journal of the Association for Information Systems, 2014, 12(8), pp. 556-584.

[5] L. Dubé, and D. Robey, "Surviving the paradox of virtual teamwork", Information Systems Journal, 2009, 19(1), pp. 3-30.

[6] J. Kotlarsky, and I. Oshri, "Social ties, knowledge sharing and successful collaboration in globally distributed system development projects", European Journal of Information Systems, 2005, 14, pp. 37-48.

[7] J. F. Nunamaker, B. A. Reinig, and R. O. Briggs, "Principles for effective virtual teamwork", Communications of the ACM, 2009, 52(4), pp. 113-117.

[8] M. Maznevski, and K. Chudoba, "Bridging Space Over Time: Global Virtual Team Dynamics and Effectiveness", Organization Science, 2000, 11(5), pp. 473-492.

[9] S. J. Best, \& B. S. Krueger, "Online Interactions and Social Capital Distinguishing Between New and Existing Ties", Social Science Computer Review, 2006, 24(4), 395410.

[10] M. T. Hansen, "The Search-Transfer Problem: The Role of Weak Ties in Sharing Knowledge across Organization Subunits", Administrative Science Quarterly, 1999, 44, pp. 82-111.

[11] D. Krackhardt, "The Strength of Strong Ties: The Importance of Philos in Organizations", In N. Nohria \& R. Eccles (eds.), Networks and Organizations: Structure, Form, and Action. Boston: Harvard Business School Press, 1992, pp. 216-239.

[12] M. Tortoriello, R. Reagans, and B. McEvily, "Bridging the Knowledge Gap: The Influence of Strong Ties, Network Cohesion, and Network Range on the Transfer of Knowledge Between Organizational Units", Organization Science, 2009, 23(4), pp. 1024-1039.

[13] A. Petróczi, Bazsó, F, and Nepusz, T. "Measuring tiestrength in virtual social networks", Connections, 2007, 27(2), pp. 39-52.

[14] M. S. Granovetter, "The Strength of Weak Ties", American Journal of Sociology, 1973, 78(6), pp. 13601380.

[15] P. Mayring, "Qualitative Content Analysis", Forum: Qualitative Social Research, 2000, 1(2).

[16] Flick, U., The SAGE Handbook of Qualitative Analysis, SAGE Publications, London, 2014.

[17] A. Dennis, R. Fuller, and J. Valacich. "Media, tasks, and communication processes: a theory of media synchronicity", MIS Quarterly, 2008, 32(3), pp. 575-600.

[18] C. Haythornthwaite, "Strong, Weak, and Latent Ties and the Impact of New Media", The Information Society, 2002, 18(5), pp. 385-401.

[19] D. Z. Levin, and R. Cross, "The Strength of Weak Ties You Can Trust: The Mediating Role of Trust in Effective Knowledge Transfer", Management Science, 50(11), pp. 1477-1490.
[20] D. Constant, L. Sproull, and S. Kiesler, "The Kindness of Strangers: The Usefulness of Electronic Weak Ties for Technical Advice", Organization Science, 1996, 7(2), 119135.

[21] C. Chiu, Y. Fan, F. Shih, and E. Wang, "Understanding knowledge sharing in virtual communities: An integration of expectancy disconfirmation and justice theories", Online Information Review, 2010, 35(1), pp. 134-153.

[22] N. Kock, and J. Nosek, "Expanding the boundaries of e-collaboration", IEEE Transactions on Professional Communication, 2005, 48(1), pp. 1-9.

[23] N. Choi, K. Palmer, and L. Horowitz, "Web 2.0 Use and Knowledge Transfer: How Social Media Technologies Can Lead to Organizational Innovation", The Electronic Journal of Knowledge Management, 2014, 12(3), pp. 176186.

[24] Y. Hung, A. Dennis, and L. Robert, "Trust in virtual teams: towards an integrative model of trust formation", Proceedings of 37th Annual Hawaii International Conference on System Sciences, 2004, pp. 1-11.

[25] A. Skjelve \& G. Rindahl, "Promoting trust between members of distributed teams", IEEE International Conference on Systems, Man and Cybernetics, 2010, pp. 1650-1658.

[26] V. Venkatesh, S. A. Brown, and H. Bala, "Bridging the Qualitative-Quantitative Divide: Guidelines for Conducting Mixed Methods Research in Information Systems," MIS Quarterly, 2013, 37(1), pp. 21-54.

[27] M. Patton, "Qualitative Evaluation and Research Methods", Sage, Beverly Hills, CA, pp. 169-186.

[28] U. Shultze, and M. Avital, "Designing interviews to generate rich data for information systems research", Information and Organization, 2011, 21(1), pp. 1-16.

[29] W. H. DeLone, and E. R. McLean, "The DeLone and McLean Model of Information Systems Success: A TenYear Update", Journal of Management Information Systems, 2003, 19(4), pp. 9-30.

[30] S. Retzer, P. Yoong, V. Hooper, "Inter-organisational knowledge transfer in social networks: A definition of intermediate ties", Information Systems Frontiers, 2012, 14(2), pp. 343-361.

[31] J. P. Onnela, J. Saramäki, J. Hyvönen, G. Szabó, D. Lazer, K. Kaski, J. Kertész, and A. L. Barabási, "Structure and tie strengths in mobile communication networks", Proceedings of the National Academy of Sciences of the United States of America, 2007, 104(18), pp. 7332-7336.

[32] P. Leonardi, "Social Media, Knowledge Sharing, and Innovation: Toward a Theory of Communication Visibility Social Media, Knowledge Sharing, and Innovation: Toward a Theory of Communication Visibility", Information Systems Research, 2014, 25(4), pp. 796-816.

[33] R. L. Daft, and R. H. Lengel, "Organizational information requirements, media richness and structural design”, Management Science, 32(5), 1986, pp. 554-571. 